\title{
Effect of dark-colored maple syrup on cell proliferation of human gastrointestinal cancer cell
}

\author{
TETSUSHI YAMAMOTO, KANTA SATO, YUIKA KUBOTA, KUNIKO MITAMURA and ATSUSHI TAGA \\ Pathological and Biomolecule Analyses Laboratory, Faculty of Pharmacy, \\ Kindai University, Higashi-Osaka, Osaka 577-8502, Japan
}

Received March 29, 2017; Accepted April 4, 2017

DOI: $10.3892 /$ br.2017.910

\begin{abstract}
Maple syrup is a natural sweetener that is commonly consumed worldwide. While maple syrup mainly comprises sucrose, it also contains phytochemicals that present various biological effects. Maple syrup is made by boiling down sap, and its color and composition vary in accordance with the sap collection season. Typically, seasonal progression is associated with darker syrup color, and antioxidant activity is proportional to the increasingly dark color. The authors previously reported that maple syrup demonstrated inhibitory effects on colorectal cancer cell growth and invasion, which correlated with darker maple syrup color. In the present study, they examined the effects of two different grades of maple syrup on gastrointestinal cancer cell proliferation, to investigate whether the dark-color maple syrup was suitable as a phytomedicine for gastrointestinal cancer treatment. Administration of dark-color maple syrup significantly inhibited gastrointestinal cancer cell growth as compared to non-treated cancer cells. Moreover, administration of dark-color maple syrup clearly inhibited protein kinase B (AKT) phosphorylation and did not impact mitogen-associated protein kinase phosphorylation. These data suggested that dark-color maple syrup may inhibit cell proliferation through suppression of AKT activation and, thus, may be suitable as a phytomedicine for gastrointestinal cancer treatment.
\end{abstract}

\section{Introduction}

Maple syrup, which is produced by boiling down sap collected from the sugar maple (Acer saccharum), is among the most commonly consumed natural sweeteners worldwide $(1,2)$. The sugar maple is distributed throughout northeastern North America, and maple syrup is mainly produced in this region (2).

Correspondence to: Dr Atsushi Taga, Pathological and Biomolecule Analyses Laboratory, Faculty of Pharmacy, Kindai University, 3-4-1 Kowakae, Higashi-Osaka, Osaka 577-8502, Japan E-mail:punk@phar.kindai.ac.jp

Key words: maple syrup, cell proliferation, gastrointestinal cancer, protein kinase $\mathrm{B}$, phytomedicine
The North American maple tree has served an important role in traditional medicine among Native Americans (3).

Maple syrup mainly comprises sucrose, but also contains various other components, such as oligosaccharides, polysaccharides, organic acids, amino acids, vitamins and minerals (1,2,4-8). Previous studies, some recent, reported that maple syrup also contains several phytochemicals, including phenolic compounds that present hypoglycemic, antioxidant, antimutagenic, anticancer, anti-inflammatory, antibiotic and anti-neurodegenerative effects (9-17). Moreover, biological effects have been noted following maple syrup consumption. In a model of type 2 diabetes mellitus (the Otsuka Long-Evans Tokushima Fatty rat), oral administration of maple syrup leads to a lower increase in plasma glucose compared to oral administration of sucrose (18). Additionally, maple syrup administration inhibits colorectal cancer cell proliferation and invasion via inhibition of protein kinase B (AKT) activation (19).

The composition and sugar content of maple sap are associated with climatic conditions of the production season (20-23). Therefore, the color, aroma and taste of maple syrup varies based on differences in growth conditions and on the season of sap collection. Maple syrup grade is primarily determined based on flavor and color, ranging from very light-colored and delicately flavored to very dark-colored and strongly flavored (2). This suggests that different grades of maple syrup could have different biological effects; however, this subject has been scarcely studied. The authors previously reported that maple syrup demonstrates an inhibitory effect on colorectal cancer cell growth and invasion, and that this anticancer effect correlates with darker maple syrup color (19).

In the present study, the authors examined the effects of different grades of maple syrup on gastrointestinal cancer cell proliferation. The two types of maple syrup that indicated the strongest and weakest anticancer effects in our previous study of colon cancer cells were used. The aim of the present work was to investigate whether the dark-color maple syrup is suitable as a phytomedicine and whether it may be useful in the development of novel anticancer drugs for gastrointestinal cancer.

\section{Materials and methods}

Materials. Chemicals and reagents of the highest grade available were purchased as follows: urea from GE Healthcare 
Life Sciences (Chalfont, UK); 3-(3-cholamidepropyl) dimethylammonio-1-propanesulphonate (CHAPS) from Wako Pure Chemical Industries, Ltd. (Osaka, Japan); and thiourea and Triton X-100 from Nacalai Tesque, Inc. (Kyoto, Japan). All other chemicals and reagents were purchased from Sigma-Aldrich; Merck KGaA (Darmstadt, Germany).

Maple syrup samples. Maple syrups were purchased at a local grocery store (Osaka, Japan) in 2015. The authors selected two maple syrups of different colors: Maple syrup A was slightly golden, and maple syrup B was very dark brown.

High-performance liquid chromatography (HPLC) analysis. To determine the carbohydrate concentrations of each maple syrup type, an LC-10Advp HPLC system was used (Shimadzu Corporation, Kyoto, Japan) equipped with a Corona Veo detector (Thermo Fisher Scientific, Inc., Waltham, MA, USA) and an Asahipak NH2P-50 4E (Shimadzu Corporation) column at room temperature $\left(\sim 23^{\circ} \mathrm{C}\right)$. The mobile phase was acetonitrile/milliQ water $(3: 1 ; \mathrm{v} / \mathrm{v})$, at a flow rate of $1 \mathrm{ml} / \mathrm{min}$. A total of $20 \mu \mathrm{l}$ sample solution was then injected, which was prepared as follows. The authors evaporated $10 \mu \mathrm{l}$ maple syrup using a Spin Dryer mini VC-15S (Taitec Corporation, Saitama, Japan). The residue was resuspended in $250 \mu \mathrm{l}$ water, and then we extracted the hydrophobic components of the maple syrup solution using ethyl acetate. The aqueous phase was ultrafiltered using an Amicon Ultra 10K device (EMD Millipore, Billerica, MA, USA) to remove high-molecular-weight components. Finally, the filtrate was diluted 1:100 in water.

Gastrointestinal cancer cell lines. The SW480 colorectal cancer cell line, KATO III gastric cancer cell line, OE33 esophageal cancer cell line, and PANC-1 pancreatic cancer cell lines were obtained from the American Type Culture Collection (Manassas, VA, USA). All cells were cultured in RPMI-1640 medium supplemented with $10 \%$ fetal bovine serum (Gibco; Thermo Fisher Scientific, Inc.) in an atmosphere containing $5 \% \mathrm{CO}_{2}$.

Cell proliferation assays. Cell lines were cultured in six-well plates at a density of $5 \times 10^{4}$ cells/well. At $24 \mathrm{~h}$ of growth, the normal medium was replaced with culture medium containing $1 \%$ (v/v) maple syrup, based on the author's previous study (19). Following 24, 48, 72 and 96 h of growth, the number of cells was counted using a Countess Automated Cell counter (Thermo Fisher Scientific, Inc.).

Protein preparation. Cells were plated at a density of $5 \times 10^{5}$ cells per $100 \mathrm{~mm}$ dish. At $24 \mathrm{~h}$ of growth, the normal medium was replaced with culture medium containing maple syrup. Following $72 \mathrm{~h}$, the cells were solubilized in urea lysis buffer (7 M urea, $2 \mathrm{M}$ thiourea, 5\% CHAPS and 1\% Triton X-100). The protein concentration was measured using the Bio-Rad Protein assay (Bio-Rad Laboratories, Inc., Hercules, CA, USA).

Western blot analysis. A total of $10 \mu \mathrm{g}$ protein was added to each well and was subjected to $10 \%$ SDS-PAGE under reducing conditions, and the separated proteins were transferred to polyvinylidene fluoride transfer membranes. Following blocking in TBS-Tween-20 (0.1\%) buffer with 5\% skim milk for $2 \mathrm{~h}$ at room temperature, the membranes were incubated at $4^{\circ} \mathrm{C}$ overnight with an anti-phospho-AKT $(1 ; 1,000$; cat. no. 4051; Cell Signaling Technology, Inc., Danvers, MA, USA), anti-phospho-p44/42 MAPK (1;1,000; cat. no. 4370; Cell Signaling Technology, Inc.), anti-phospho-SAPK/JNK (1;1,000; cat. no. 4668; Cell Signaling Technology, Inc.), or anti-phospho-p38 MAPK antibody $(1 ; 1,000$; cat. no. 4511; Cell Signaling Technology, Inc.). Then the membranes were washed and incubated with horseradish peroxidase-conjugated anti-rabbit or anti-mouse IgG antibody (American Qualex, San Clemente, CA, USA). Following washing, the blots were visualized using SuperSignal West Dura Extended Duration substrate (Thermo Fisher Scientific, Inc.), and bands were detected using a myECL Imager system (version 2.0; Thermo Fisher Scientific, Inc.). Next, the same membranes were re-probed with anti- $\beta$-actin (Sigma-Aldrich; Merck KGaA), anti-AKT, anti-p44/42 MAPK (Erk1/2), anti-SAPK/JNK or anti-p38 MAPK antibody (Cell Signaling Technology, Inc.) to confirm equal loading of the proteins. All western blot analyses were performed in triplicate.

Statistical analysis. All data are presented as the mean \pm standard error of the mean. The data were analyzed using one-way analysis of variance followed by Dunnett's test. $\mathrm{P} \leq 0.05$ was considered to indicate a statistically significant difference. Computations were performed using GraphPad Prism software (version 5; GraphPad Software, La Jolla, CA, USA).

\section{Results}

Carbohydrate concentrations in the two maple syrup types. HPLC analysis revealed that both maple syrup A and B contained abundant sucrose, and smaller concentrations of glucose and fructose (Fig. 1A and B). Sucrose concentration did not significantly differ between the two types of maple syrup. Compared to maple syrup A, maple syrup B contained significantly higher concentrations of glucose and fructose $(\mathrm{P}<0.05$; Table I).

Effect of maple syrup on gastrointestinal cancer cell growth. Compared to non-treated control cells, administration of maple syrup B significantly inhibited the growth of SW480 ( $\mathrm{P}<0.01$; Fig. 2A; 72 and 96 h), KATO III ( $<<0.05$; Fig. 2B; 72 and $96 \mathrm{~h}$ ), OE33 ( $<<0.01$; Fig. 2C; $96 \mathrm{~h})$, and PANC-1 $(\mathrm{P}<0.01$; Fig. 2D; 48 and $72 \mathrm{~h}$ ) cells. Administration of maple syrup A also significantly inhibited the growth of SW480 (P<0.01; Fig. 2A; 96 h) and PANC-1 (P<0.05; Fig. 2D; 48 h) cells as compared to non-treated control cells.

Effects of maple syrup on signaling pathways in gastrointestinal cancer cells. To determine which signaling pathway was affected by maple syrup administration, the authors examined the phosphorylation of ERK, JNK, p38 and AKT, which serve important roles in gastrointestinal cancer cell proliferation. Administration of maple syrup B visibly inhibited AKT phosphorylation in all tested gastrointestinal cancer cell lines, as compared to non-treated cells and cells treated with maple syrup A (Fig. 3A-D). Conversely, administration of 
Table I. Maple syrup color and carbohydrate concentrations.

\begin{tabular}{lcccc}
\hline Variables & Color & Sucrose $(\mathrm{g} / 100 \mathrm{ml})$ & Glucose $(\mathrm{g} / 100 \mathrm{ml})$ & Fructose $(\mathrm{g} / 100 \mathrm{ml})$ \\
\hline Maple syrup A & Slightly golden & $53.02 \pm 2.20$ & $0.28 \pm 0.03$ & $0.82 \pm 0.05$ \\
Maple syrup B & Very dark brown & $49.83 \pm 0.16$ & $1.91 \pm 0.05^{\mathrm{a}}$ & $2.38 \pm 0.02^{\mathrm{a}}$ \\
\hline
\end{tabular}

${ }^{\text {ap }}<0.05$ vs. maple syrup A.

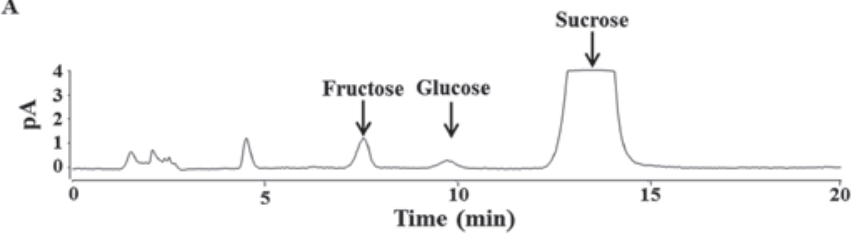

B

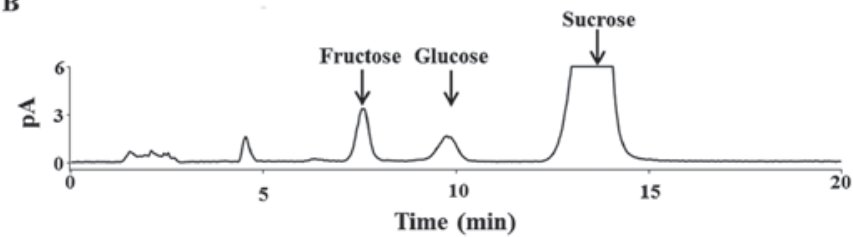

C

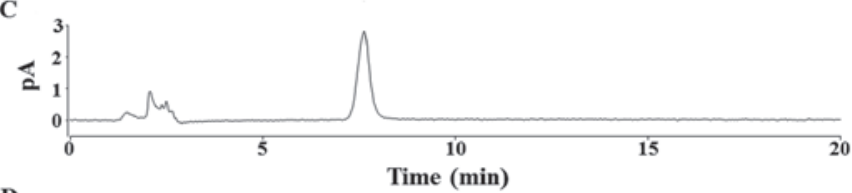

D

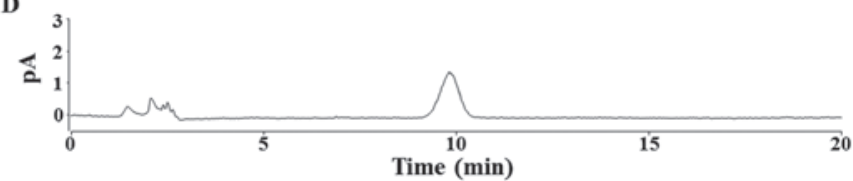

E

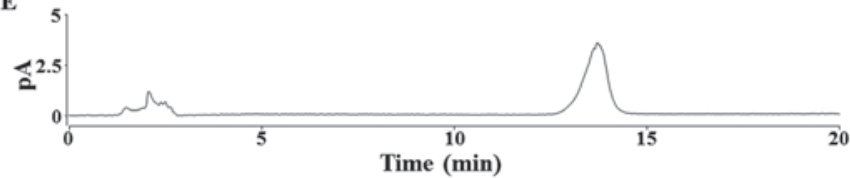

Figure 1. High-performance liquid chromatography chromatograms of (A) maple syrup A, (B) maple syrup B, (C) $50 \mu \mathrm{M}$ fructose, (D) $50 \mu \mathrm{M}$ glucose and (E) $62.5 \mu \mathrm{M}$ sucrose.

maple syrup B inhibited phosphorylation of ERK1/2 in OE33 cells (Fig. 3C). Maple syrup administration did not influence MAPK activation in any other tested gastrointestinal cancer cell lines.

\section{Discussion}

In the present study, the authors examined the inhibitory effects of two different grades of maple syrup on cell proliferation of esophageal cancer (OE33), gastric cancer (KATO III), colon cancer (SW480) and pancreatic cancer (PANC-1) cell lines. As carbohydrates are the primary components of maple syrup, the authors first examined the carbohydrate composition of each maple syrup type to determine a suitable maple syrup dosage to avoid cytotoxic effects from excessive sucrose. HPLC analysis of each maple syrup type presented three peaks, corresponding to sucrose, glucose and fructose, with sucrose being the primary component, which is consistent with previous reports (Fig. 1A and B) $(1,24)$. Maple syrup B had significantly higher concentrations of glucose and fructose, compared to maple syrup A (Table I). Conversely, sucrose concentration was similar in both grades of maple syrup (Table I), and also similar to the authors' previous analysis of maple syrup that was purchased in a different year (19). Thus, for further studies, the authors used the same dose of maple syrup as was administered in previous reports, which did not induce cytotoxicity due to high sucrose concentration (19).

It was identified that administration of maple syrup B led to a significant decrease in the cell growth rate of all examined gastrointestinal cancer cells compared to non-treated control cells (Fig. 2). Additionally, administration of maple syrup A led to significant inhibition of cell growth among colon and pancreatic cancer cells compared to non-treated control cells (Fig. 2A and D). Interestingly, the dark-color maple syrup had an anti-proliferative effect on all investigated gastrointestinal cancer cells, even though it contained a higher amount of glucose than the golden-color maple syrup. In contrast, the golden-color maple syrup did not significantly inhibit cell proliferation for all investigated gastrointestinal cancer cells, but it did present a tendency to suppress cell proliferation. These data suggested that the maple syrup contained an active ingredient that suppressed cancer cell proliferation, and that the amount of this active ingredient increased with darkening syrup color.

Following this, the authors examined how maple syrup affected the phosphorylation status of ERK, JNK, p38 and AKT, which each serve important roles in cancer cell proliferation. Administration of dark-color maple syrup inhibited AKT activation in colon cancer cells, consistent with previous data (19). Moreover, it demonstrated similar inhibitory effects in the other gastrointestinal cancer cells used in this study. Maple syrup contains various phenolic compounds, including lignans and coumarins. Such phenolic compounds have been previously reported to present inhibitory effects on AKT activation in cancer cells (25-31). The present data suggested that dark-color maple syrup may contain larger amounts of bioactive compounds, such as phenolic compounds.

On the other hand, administration of dark-color maple syrup did not affect MAPK activation, except for an inhibitory effect on ERK activation in esophageal cancer cells (Fig. 3). Notably, despite suppression of two signaling pathways related to cell proliferation, the inhibitory effect on esophageal cancer cell proliferation following administration of dark-color maple syrup was to a lesser extent than the effect observed in other gastrointestinal cancer cells. These data suggested that administration of dark-color maple syrup in esophageal cancer cells may have other effects, such as inhibition of cancer cell 
A

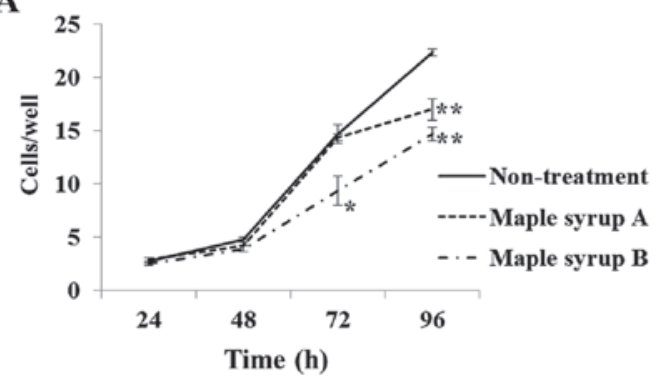

C

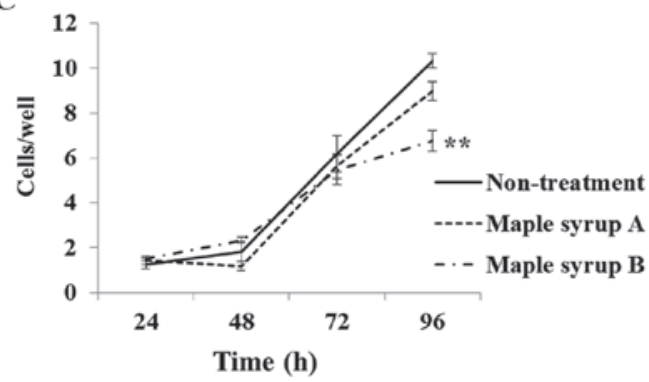

B

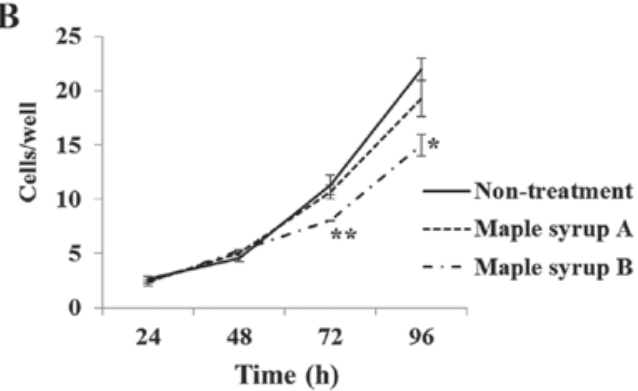

D

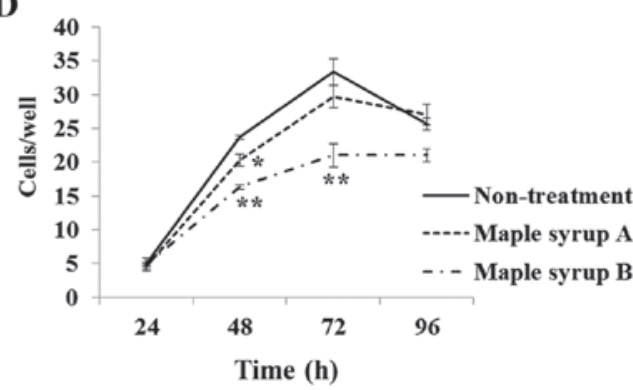

Figure 2. Effects of maple syrup on gastrointestinal cancer cell proliferation. Compared to non-treated controls, administration of maple syrup B significantly inhibited cell proliferation of (A) SW480 (72 and 96 h), (B) KATO III (72 and 96 h), (C) OE33 (96 h) and (D) PANC-1 (48 and 72 h) cells. Administration of maple syrup A also inhibited proliferation of (A) SW480 (96 h) and (D) PANC-1 (48 h) cells compared to non-treated cells. ${ }^{*} \mathrm{P}<0.05$, ${ }^{* * *} \mathrm{P}<0.01$ vs. non-treatment.

A

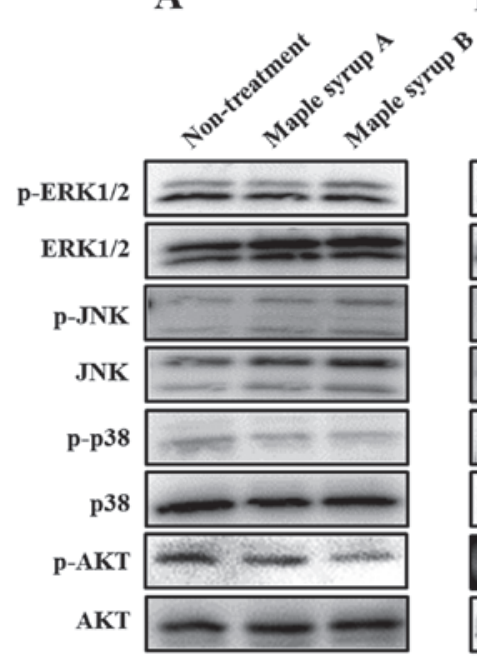

B

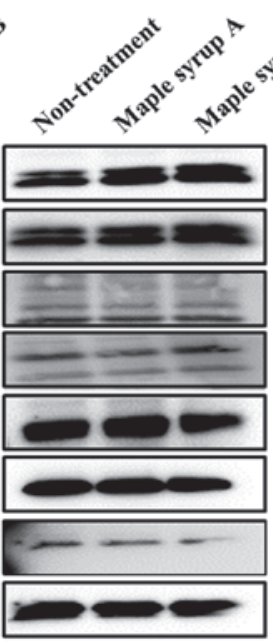

C

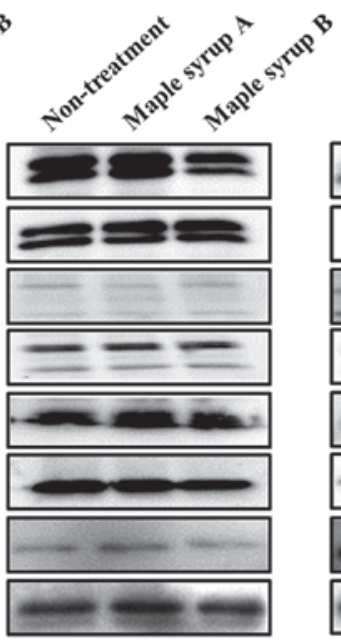

D

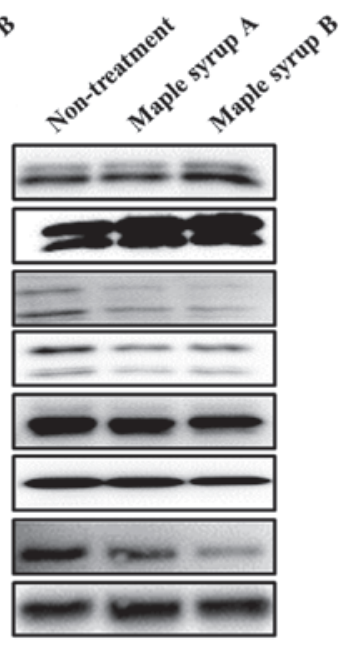

Figure 3. Effects of maple syrup on the mitogen-associated protein kinase and AKT signaling pathways of gastrointestinal cancer cell lines (A) SW480, (B) KATO III, (C) OE33 and (D) PANC-1. AKT, protein kinase B.

invasion, particularly since a previous study of the authors demonstrated that administration of dark-color maple syrup inhibited colon cancer cell invasion (19).

The current results indicated that dark-color maple syrup had anti-cancer effects in upper digestive tract cancer cell lines, such as esophageal and gastric cancer. This suggests that routine intake of dark-color maple syrup could potentially suppress the progression of esophageal and gastric cancer. Moreover, administration of dark-color maple syrup presented anti-cancer effects in pancreatic cancer cells, which is a type of cancer that is refractory to current treatments. Therefore, the bioactive compounds in dark-color maple syrup may be useful in the development of novel anti-cancer drugs for treatment of gastrointestinal cancer as well as refractory cancers.
Further studies are required to clarify the optimal dosage and administration method for using dark-color maple syrup as a phytomedicine for cancer treatment. Future studies are also warranted to identify the bioactive compounds in dark-color maple syrup that are responsible for repressing cancer cell proliferation through inhibition of the AKT signaling pathway.

In conclusion, the present data demonstrated that dark-color maple syrup reduced gastrointestinal cancer cell growth through inhibition of the AKT signaling pathway. These findings suggested that dark-color maple syrup may be useful as phytomedicine, potentially preventing cancer progression. The present data may also be useful for future development of novel anti-cancer drugs for gastrointestinal cancer treatment with fewer adverse effects than traditional chemotherapy. 


\section{Acknowledgements}

The present study was supported in part by a MEXT (Ministry of Education, Culture, Sports. Science and Technology)-Supported Program for the Strategic Research Foundation at Private Universities (grant no. S1411037), and by a Grant-in-Aid for Scientific Research from the Japan Society for the Promotion of Science to T.Y. (grant no. 15K09054).

\section{References}

1. Ball DW: The chemical composition of maple syrup. J Chem Educ 84: 1647-U1646, 2007.

2. Perkins TD and van den Berg AK: Maple syrup-production, composition, chemistry, and sensory characteristics. Adv Food Nutr Res 56: 101-143, 2009.

3. Arnason T, Hebda RJ and Johns T: Use of plants for food and medicine by Native Peoples of eastern Canada. Can J Bot 59: 2189-2325, 1981

4. Davison RM and Young H: Abscisic-acid content of xylem sap. Planta 109: 95-98, 1973

5. Taga A, Sato A, Suzuki K, Takeda M and Kodama S: Simple determination of a strongly aromatic compound, sotolon, by capillary electrophoresis. J Oleo Sci 61: 45-48, 2012.

6. Taga A and Kodama S: Analysis of reducing carbohydrates and fructosyl saccharides in maple syrup and maple sugar by $\mathrm{CE}$. Chromatographia 75: 1009-1016, 2012.

7. Storz G, Darvill AG and Albersheim P: Characterization of polysaccharides isolated from maple syrup. Phytochemistry 25: 437-441, 1986

8. Sun J, Ma H, Seeram NP and Rowley DC: Detection of Inulin, a Prebiotic Polysaccharide, in Maple Syrup. J Agric Food Chem 64: 7142-7147, 2016.

9. Apostolidis E, Li LY, Lee C and Seeram NP: In vitro evaluation of phenolic-enriched maple syrup extracts for inhibition of carbohydrate hydrolyzing enzymes relevant to type 2 diabetes management. J Funct Foods 3: 100-106, 2011.

10. Legault J, Girard-Lalancette K, Grenon C, Dussault C and Pichette A: Antioxidant activity, inhibition of nitric oxide overproduction, and in vitro antiproliferative effect of maple sap and syrup from Acer saccharum. J Med Food 13: 460-468, 2010.

11. González-Sarrías A, Li L and Seeram NP: Effects of maple (Acer) plant part extracts on proliferation, apoptosis and cell cycle arrest of human tumorigenic and non-tumorigenic colon cells. Phytother Res 26: 995-1002, 2012.

12. Li L and Seeram NP: Maple syrup phytochemicals include lignans, coumarins, a stilbene, and other previously unreported antioxidant phenolic compounds. J Agric Food Chem 58: 11673-11679, 2010.

13. Theriault M, Caillet S, Kermasha S and Lacroix M: Antioxidant, antiradical and antimutagenic activities of phenolic compounds present in maple products. Food Chem 98: 490-501, 2006.

14. Hawco CLA, Wang Y, Taylor M and Weaver DF: A maple syrup extract prevents $\beta$-amyloid aggregation. Can J Neurol Sci 43 : 198-201, 2016.

15. Aaron C, Beaudry G, Parker JA and Therrien M: Maple Syrup Decreases TDP-43 Proteotoxicity in a Caenorhabditis elegans Model of Amyotrophic Lateral Sclerosis (ALS). J Agric Food Chem 64: 3338-3344, 2016.

16. Maisuria VB, Hosseinidoust Z and Tufenkji N: Polyphenolic extract from maple syrup potentiates antibiotic susceptibility and reduces biofilm formation of pathogenic bacteria. Appl Environ Microbiol 81: 3782-3792, 2015.
17. Ma H, DaSilva NA, Liu W, Nahar PP, Wei Z, Liu Y, Pham PT, Crews R, Vattem DA, Slitt AL, et al: Effects of a Standardized Phenolic-Enriched Maple Syrup Extract on $\beta$-Amyloid Aggregation, Neuroinflammation in Microglial and Neuronal Cells, and $\beta$-Amyloid Induced Neurotoxicity in Caenorhabditis elegans. Neurochem Res 41: 2836-2847, 2016.

18. Nagai $\mathrm{N}$, Ito $\mathrm{Y}$ and Taga A: Comparison of the enhancement of plasma glucose levels in type 2 diabetes Otsuka Long-Evans Tokushima Fatty rats by oral administration of sucrose or maple syrup. J Oleo Sci 62: 737-743, 2013.

19. Yamamoto T, Uemura K, Moriyama K, Mitamura K and Taga A: Inhibitory effect of maple syrup on the cell growth and invasion of human colorectal cancer cells. Oncol Rep 33: 1579-1584, 2015

20. Kim YT and Leech RH: Effects of climatic conditions on sap flow in sugar maple. For Chron 61: 303-307, 1985.

21. Marvin JW and Erickson RO: A statistical evaluation of some of the factors responsible for the flow of sap from the sugar maple. Plant Physiol 31: 57-61, 1956.

22. Plamondon AP and Bernier PY: Modélisation de la coulée de l'érable à sucre (Acersaccharum Marsh.) à partir d'éléments météorologiques. Can J Res 10: 152-157, 1980.

23. Houle D, Paquette A, Côté B, Logan T, Power H, Charron I and Duchesne L: Impacts of Climate Change on the Timing of the Production Season of Maple Syrup in Eastern Canada. PLoS One 10: $\mathrm{e} 0144844,2015$.

24. Stuckel JG and Low NH: The chemical composition of 80 pure maple syrup samples produced in North America. Food Res Int 29: 373-379, 1996.

25. Huang JS, Yao CJ, Chuang SE, Yeh CT, Lee LM, Chen RM, Chao WJ, Whang-Peng J and Lai GM: Honokiol inhibits sphere formation and xenograft growth of oral cancer side population cells accompanied with JAK/STAT signaling pathway suppression and apoptosis induction. BMC Cancer 16: 245 , 2016.

26. Jiang Y, Zhang Q, Bao J, Du CH, Wang J, Tong Q and Liu C: Schisandrin B suppresses glioma cell metastasis mediated by inhibition of mTOR/MMP-9 signal pathway. Biomed Pharmacother 74: 77-82, 2015.

27. Hyun S, Kim MS, Song YS, Bak Y, Ham SY, Lee DH, Hong J and Yoon DY: Peroxisome proliferator-activated receptor-gamma agonist 4-O-methylhonokiol induces apoptosis by triggering the intrinsic apoptosis pathway and inhibiting the PI3K/Akt survival pathway in SiHa human cervical cancer cells. J Microbiol Biotechnol 25: 334-342, 2015.

28. Zilla MK, Nayak D, Amin H, Nalli Y, Rah B, Chakraborty S, Kitchlu S, Goswami A and Ali A: 4'-Demethyl-deoxypodophyllotoxin glucoside isolated from Podophyllum hexandrum exhibits potential anticancer activities by altering Chk-2 signaling pathway in MCF-7 breast cancer cells. Chem Biol Interact 224: 100-107, 2014.

29. Jeon YJ, Cho JH, Lee SY, Choi YH, Park H, Jung S, Shim JH and Chae JI: Esculetin Induces Apoptosis Through EGFR/PI3K/Akt Signaling Pathway and Nucleophosmin Relocalization. J Cell Biochem 117: 1210-1221, 2016

30. Hsieh CJ, Kuo PL, Hou MF, Hung JY, Chang FR, Hsu YC, Huang YF, Tsai EM and Hsu YL: Wedelolactone inhibits breast cancer-induced osteoclastogenesis by decreasing Akt/mTOR signaling. Int J Oncol 46: 555-562, 2015.

31. Kim WJ, Lee MY, Kim JH, Suk K and Lee WH: Decursinol angelate blocks transmigration and inflammatory activation of cancer cells through inhibition of PI3K, ERK and NF-kappaB activation. Cancer Lett 296: 35-42, 2010. 